\title{
Evaluation of injection-site-related adverse events with galcanezumab: a post hoc analysis of phase 3 studies in participants with migraine
}

Virginia L. Stauffer ${ }^{1 *}$, Shufang Wang ${ }^{1}$, Jo Bonner ${ }^{2}$, ByungKun Kim ${ }^{3}$, Rohit Bhandari ${ }^{4}$, Kathleen A. Day ${ }^{1}$ and Angelo Camporeale ${ }^{5}$

\begin{abstract}
Background: Injection-site reactions have been reported with biologicals. In this post hoc analysis of Phase 3 studies in participants with migraine, we provide a comprehensive overview and detailed summary of injection-site reaction with galcanezumab.

Methods: Data were obtained from two randomised clinical studies in participants with episodic migraine (EVOLVE1 and EVOLVE-2), one randomised study in participants with chronic migraine (REGAIN) and one open-label study (Study CGAJ) in participants with episodic or chronic migraine. The injection-site reactions were measured for two different cohorts: 1) six-month double-blind treatment phase in the EVOLVE-1 and EVOLVE-2 studies and threemonth double-blind treatment phase in the REGAIN study, where participants received placebo and galcanezumab (placebo-controlled analysis set); 2) three month double-blind (Month 0 to Month 3; 1:1:placebo:galcanezumab) +9 months open-label extension phase (Month 3 to Month 12) of REGAIN and twelve month open-label phase of Study CGAJ, where participants received only galcanezumab (galcanezumab exposure analysis set).

Results: A total of 477 participants in the placebo-controlled analysis set (galcanezumab $240 \mathrm{mg}, 166 / 730$ [22.7\%]; galcanezumab 120 mg, 128/705 [18.2\%]; placebo, 183/1451 [12.6\%]) reported at least one injection-site reaction. Most of the injection-site reactions were reported as injection-site pain, unspecified injection-site reaction, injection-site erythema, and injection-site pruritus. The incidence of injection-site pain was highest among all reported injection-site reactions and were reported with similar frequency by participants receiving galcanezumab (galcanezumab $120 \mathrm{mg}, 10.1 \%$; galcanezumab $240 \mathrm{mg}, 11.6 \%$ ) and placebo (9.5\%) and was the most common injection-site reaction reported within 60 min of injection ( $86 \%$ of participants). The frequency of unspecified injection-site reaction, injection-site erythema and injection-site pruritus was significantly $(P<0.001)$ higher in participant receiving galcanezumab versus placebo. In the galcanezumab exposure analysis set participants received up to 12 doses and the frequency of injection-site reactions reported for both doses combined was $21.8 \%$. The reporting of injection-site reactions did not increase with (Continued on next page)
\end{abstract}

\footnotetext{
* Correspondence: vstauffer@lilly.com

${ }^{1}$ Lilly Research Laboratories, Lilly Corporate Center, Indianapolis, IN, USA

Full list of author information is available at the end of the article
}

(c) The Author(s). 2020 Open Access This article is licensed under a Creative Commons Attribution 4.0 International License, which permits use, sharing, adaptation, distribution and reproduction in any medium or format, as long as you give appropriate credit to the original author(s) and the source, provide a link to the Creative Commons licence, and indicate if changes were made. The images or other third party material in this article are included in the article's Creative Commons. licence, unless indicated otherwise in a credit line to the material. If material is not included in the article's Creative Commons licence and your intended use is not permitted by statutory regulation or exceeds the permitted use, you will need to obtain permission directly from the copyright holder. To view a copy of this licence, visit http://creativecommons.org/licenses/by/4.0/ The Creative Commons Public Domain Dedication waiver (http://creativecommons.org/publicdomain/zero/1.0/) applies to the data made available in this article, unless otherwise stated in a credit line to the data. 
(Continued from previous page)

the number of doses received. No ISR-related serious adverse events were reported in both the placebocontrolled and galcanezumab exposure analysis sets.

Conclusions: The most common adverse event of galcanezumab is injection-site reactions. However, these events were generally mild-to-moderate in severity, non-serious, resolved spontaneously, and discontinuations due to injection-site reactions were low (1\%).

Keywords: Injection-site reaction, Monoclonal antibody, Migraine, Physiological, Formulation factors, Calcitonin gene-related peptide

\section{Background}

People with migraine have been shown to have elevated blood levels of calcitonin gene-related peptide (CGRP) and targeting the CGRP pathway using antibodies has been demonstrated to be effective in preventing migraine attacks [1-4]. Three such biological therapies including galcanezumab have been approved as preventive treatments for migraine [5]. Galcanezumab, erenumab and fremanezumab are administered subcutaneously, and adverse events (AEs) related to injection-sites were the most frequently reported AEs in their respective Phase 3 programs [6-8]. Injection-site reactions are local skin reactions occurring after an injection and include injection-site pain, erythema, pruritus and induration [6-8]. The aetiology of injection-site-related adverse events maybe multifocal, ranging from immunological to non-immunological factors including injection volume, temperature, $\mathrm{pH}$, speed of injection, needle size, and injection excipients $[9,10]$.

Efficacy of galcanezumab was demonstrated in pivotal phase 3 studies in patients with episodic or chronic migraine $[4,11-13]$. In the phase 3 studies, injection-site reactions were the most frequently reported AEs with galcanezumab treatment $[4,11,12,14]$.

Therefore, in this post hoc analysis of Phase 3 migraine studies of galcanezumab, we provide a comprehensive overview and detailed summary of injection-site reactions.

\section{Methods}

Data were obtained from two randomised clinical studies in participants with episodic migraine (EVOLVE-1, clini caltrials.gov identifier: NCT02614183, https://clinicaltrials. gov/ct2/show/NCT02614183; EVOLVE-2, clinicaltrials. gov identifier: NCT02614196, https://clinicaltrials.gov/ct2/ show/NCT02614196), one randomised study in participants with chronic migraine (REGAIN, clinicaltrials.gov identifier: NCT02614261, https://clinicaltrials.gov/ct2/ show/NCT02614261) and one open-label study (CGAJ, clinicaltrials.gov identifier: NCT02614287, https://clinical trials.gov/ct2/show/NCT02614287) in participants with episodic or chronic migraine. All clinical trials were first posted on 25 November 2015, and before enrolling the first patient. The EVOLVE and REGAIN studies were designed to study efficacy and safety of galcanezumab, and the open-label Study CGAJ was designed primarily to assess long-term safety. The study designs of all four studies have been previously published $[4,11,12,14]$.

Briefly, EVOLVE-1 and EVOLVE-2 studies had a 6month double-blind (DB) treatment phase wherein participants were randomised 2:1:1 to receive monthly placebo, galcanezumab $120 \mathrm{mg}$ or galcanezumab $240 \mathrm{mg}$. The REGAIN study had a 3-month DB treatment phase, wherein participants were randomised 2:1:1 to receive monthly placebo, galcanezumab $120 \mathrm{mg}$, or galcanezumab $240 \mathrm{mg}$. The DB phase was followed by an optional nine month openlabel extension (OLE) phase wherein either galcanezumab $120 \mathrm{mg}$ or galcanezumab $240 \mathrm{mg}$ was administered based on the investigator's discretion. Study CGAJ comprised a 12-month open-label (OL) phase wherein participants were randomised $1: 1$ to receive galcanezumab $120 \mathrm{mg}$ or galcanezumab $240 \mathrm{mg}$. All four studies had a four-month safety follow-up period immediately after the treatment phase.

In the EVOLVE and REGAIN studies, study site personnel administered the injections using prefilled syringes. In Study CGAJ, injections were self-administered by participant or caregiver using prefilled syringes for up to 9 months, and then participants were switched to self-administer galcanezumab using an autoinjector [15]. Notably the autoinjector was not available for administration at the time of study initiation and was available approximately 1 year after the start of the study. As such all participants in Study CGAJ used an autoinjector from approximately Month 10 onwards. All participants and caregivers in the Study CGAJ who continued the treatment were trained to use the autoinjector before switching at Month 9 [15].

\section{Evaluation of injection site reactions}

Two cohorts from the integrated analysis sets of four studies (EVOLVE-1, EVOLVE-2, REGAIN and CGAJ) were analysed. These studies included: 1) all participants from the six-month $\mathrm{DB}$ treatment phase in the EVOLVE-1 and EVOLVE-2 studies and three-month DB treatment phase in the REGAIN study (placebo-controlled analysis set); 2) galcanezumab-treated participants from the DB + OLE phase of REGAIN, Month 0 to 
Month 12, and OL phase of Study CGAJ, Month 0 to Month 12, where participants received galcanezumab (galcanezumab exposure analysis set). Of note, participants who were randomized to placebo in the REGAIN study received up to 9 monthly doses of galcanezumab and participants who were randomized to galcanezumab received up to 12 monthly doses of galcanezumab.

The injection-site reactions are treatment-emergent adverse events (TEAEs) that occurred or worsened at any time after treatment initiation and were coded based on the Medical Dictionary for Regulatory Activities (https://www.med dra.org/). The injection-site reactions (plural) refer to the high-level grouping of different preferred terms (PT) used to describe various injection-site reactions. The reported verbatim terms collected during the study was mapped to a PT that best characterised the reported adverse event at the injection-site, such as "pain", "erythema", "rash" and "swelling". When the site recorded the event as "injection-site reaction" the event was mapped to an unspecified PT of injection-site reaction (singular). An injection-site adverse event follow-up form was used to further characterise the reported unspecified event of injection-site reaction.

\section{Statistical analysis}

The placebo-controlled analysis set was used to evaluate injection-site reactions for up to six-month of DB treatment and the galcanezumab exposure analysis set was used to evaluate injection-site reactions up to 12 months of galcanezumab treatment.

For both analysis sets, the number of participants with injection-site reactions, discontinuations due to AEs, and serious AEs (SAEs) were summarized. For the placebocontrolled analysis set, inferential statistics was provided. Treatment comparisons were evaluated using the CochranMantel-Haenszel test stratified by study. In addition, patients with injection-site reactions were summarized by maximum severity as "mild", "moderate" or "severe". The reaction timings from the last injection to the occurrence of injection-site reactions were summarised as follows: immediate reaction (<60 $\mathrm{min}$ ); acute reaction ( 1 to $6 \mathrm{~h}$ ); delayed reaction (classified as injection-site reactions post $6 \mathrm{~h}$ on the day of the injection up to 14 days after the day of injection); and reactions $\geq 14$ days. The duration of injection-site reactions were also summarized.

For the galcanezumab exposure data set, to determine if injection-site reactions excluding pain were reported more frequently with repeated doses of galcanezumab, the number of TEAEs were assessed by the total number of doses received.

\section{Results}

\section{Demographics}

All 3156 participants from the EVOLVE-1, EVOLVE-2 and REGAIN studies were included in this post hoc analysis (placebo, $n=1451$; galcanezumab $120 \mathrm{mg}, n=$ 840; galcanezumab $240 \mathrm{mg}, n=865)$. In this integrated analysis set of EVOLVE-1, EVOLVE-2 and REGAIN studies, participants were predominantly women (placebo, 1237/1451 [85.3\%]; galcanezumab $120 \mathrm{mg}$, 599/705 [85.0\%]; galcanezumab $240 \mathrm{mg}, 609 / 730$ [83.4\%]), with a mean age of $\sim 41$ years (Table 1 ). On an average, participants were diagnosed as having migraine $\sim 20$ years prior to study enrolment. Baseline demographics of race, region and comorbid conditions were similar across treatment groups. The most common comorbid conditions (occurring in $>10 \%$ of all participants), included seasonal allergy, drug hypersensitivity, insomnia, anxiety, depression, and back pain.

In Study CGAJ, participants were predominantly women (galcanezumab $120 \mathrm{mg}, 110 / 135$ [81.5\%]; galcanezumab $240 \mathrm{mg} \mathrm{113/135}$ [83.7\%]), with a mean age of $\sim 42$ years (mean [SD]: galcanezumab $120 \mathrm{mg}, 40.2$ [11.7]; galcanezumab $240 \mathrm{mg}, 43.7$ [11.0]) and a diagnosis of migraine of 20 years prior to study enrolment (mean [SD]: galcanezumab $120 \mathrm{mg}, 20.2$ [12.4]; galcanezumab $240 \mathrm{mg}$, $21.3[12.5])$. The detailed demographics are published elsewhere [14].

\section{Injection-site reactions during double-blind phase of EVOLVE-1, EVOLVE-2 and REGAIN studies}

During the DB treatment phase of EVOLVE-1, EVOLVE-2 and REGAIN studies, 477 (477/2886, 16.5\%) participants reported at least one injection-site reaction (galcanezumab $240 \mathrm{mg}, 166 / 730$ [22.7\%]; galcanezumab $120 \mathrm{mg}, 128 / 705$ [18.2\%]; placebo, $183 / 1451[12.6 \%])$. The injection-site reactions were significantly higher $(P \leq 0.001)$ with galcanezumab $240 \mathrm{mg}$ or $120 \mathrm{mg}$ compared with placebo (Table 2). The most commonly $(\geq 2 \%)$ observed injection-site reactions were injection-site pain, unspecified injection site reaction, injection-site erythema, and injection-site pruritus. These were reported by higher proportion of participants in galcanezumab $240 \mathrm{mg}$ and $120 \mathrm{mg}$ groups compared with placebo (Table 2).

Of the 67 galcanezumab-treated participants who reported an unspecified injection site reaction (galcanezumab $120 \mathrm{mg}, 22 / 705$ [3.1\%]; galcanezumab $240 \mathrm{mg}$, 45/ $730[6.2 \%]$ ), all participants completed at least one follow-up form which was used to further characterise the reported unspecified injection site reaction. Of these 67 participants, $59.1 \%(13 / 22)$ participants on galcanezumab $120 \mathrm{mg}$ reported itching, rash or redness, and injection-site hardening. Itching, rash or redness, and injection-site hardening were reported by $60.0 \%(27 / 45)$, $84.4 \%(38 / 45)$, and $44.4 \%(20 / 45)$ participants on galcanezumab $240 \mathrm{mg}$, respectively.

Among patients who reported injection-site reactions, most reported injection-site reactions of mild-to-moderate severity (Table 3). No injection-site reactions were reported 
Table 1 Baseline demographics and comorbid conditions in EVOLVE-1, EVOLVE-2 and REGAIN studies

\begin{tabular}{|c|c|c|c|}
\hline & Placebo $(N=1451)$ & GMB $120 \mathrm{mg}(N=705)$ & GMB $240 \mathrm{mg}(N=730)$ \\
\hline \multicolumn{4}{|l|}{ Demographics } \\
\hline Age, mean years, (SD) & $41.8(11.6)$ & $40.8(11.5)$ & $40.4(11.9)$ \\
\hline Gender (female), n (\%) & $1237(85.3)$ & $599(85.0)$ & $609(83.4)$ \\
\hline \multicolumn{4}{|l|}{ Race, n (\%) } \\
\hline White & $1112(76.6)$ & $545(77.3)$ & ${ }^{a} 555(76.1)$ \\
\hline Black or African American & $117(8.1)$ & $53(7.5)$ & ${ }^{a} 59(8.1)$ \\
\hline Asian & $89(6.1)$ & $48(6.8)$ & ${ }^{a} 42(5.8)$ \\
\hline American Indian or native Alaska & $24(1.7)$ & $10(1.4)$ & ${ }^{\mathrm{a}} 16(2.2)$ \\
\hline Native Hawaiian or other pacific islander & $2(0.1)$ & 0 & ${ }^{\mathrm{a}} 4(0.6)$ \\
\hline Multiple & $107(7.4)$ & $49(7.0)$ & ${ }^{a} 53(7.3)$ \\
\hline \multicolumn{4}{|l|}{ Region, n (\%) } \\
\hline North America & $977(67.3)$ & $472(67.0)$ & $496(68.0)$ \\
\hline Europe & $262(18.1)$ & $126(17.9)$ & $131(18.0)$ \\
\hline Other & $212(14.6)$ & $107(15.2)$ & $103(14.1)$ \\
\hline Years since migraine diagnosis, mean (SD) & $21.1(12.7)$ & $20.7(12.5)$ & $19.6(12.3)$ \\
\hline \multicolumn{4}{|l|}{$\begin{array}{l}\text { Comorbid conditions that occurred in }>10 \% \\
\text { of all participants, } n(\%)\end{array}$} \\
\hline Seasonal allergy & $307(21.2)$ & $158(22.4)$ & $122(16.7)$ \\
\hline Drug hypersensitivity & $247(17.0)$ & $123(17.5)$ & $128(17.5)$ \\
\hline Insomnia & $165(11.4)$ & 89 (12.6) & $78(10.7)$ \\
\hline Anxiety & $166(11.4)$ & $82(11.6)$ & $81(11.1)$ \\
\hline Depression & $181(12.5)$ & $84(11.9)$ & 79 (10.8) \\
\hline Back pain & $151(10.4)$ & $62(8.8)$ & $75(10.3)$ \\
\hline
\end{tabular}

Abbreviations: GMB galcanezumab, ISR injection-site reaction, $N$ number of participants in the intent-to-treat population, $n$ number of participants within each specific category, $S D$ standard deviation

${ }^{\mathrm{a}} \mathrm{N}=729$

Note: All values are for injection-site reactions during the double-blind treatment phase from study start up to 6 months for EVOLVE-1 and EVOLVE-2 and up to 3 months for REGAIN

as SAEs. Overall seven participants discontinued due to injection-site reactions (galcanezumab $120 \mathrm{mg}, n=2$; galcanezumab $240 \mathrm{mg}, n=5$ ). Among these participants, four participants discontinued due to moderate unspecified injection-site reaction (galcanezumab $120 \mathrm{mg}, 1 / 705$ [0.1\%]; galcanezumab $240 \mathrm{mg}, 3 / 730$ [0.4\%]). The remaining three patients discontinued due to moderate injection-site pain (galcanezumab $240 \mathrm{mg}, 1 / 1451$ [0.1\%]), severe injection-site erythema (galcanezumab $120 \mathrm{mg}, 1 / 705$ [0.1\%]), and moderate injection-site swelling (galcanezumab $240 \mathrm{mg}, 1 / 730$ [0.1\%]) Table 3.

Injection-site pain was the most common immediate injection-site reaction reported within $60 \mathrm{~min}$ of injection) and was observed in approximately $86 \%$ of participants reporting injection-site pain (Table 4). Majority of unspecified-injection-site reaction (placebo, 100.0\%; galcanezumab, 88.0\%), injection-site erythema (placebo, 95.0\%; galcanezumab, 79.0\%) and injection-site pruritus (placebo, 100\%; galcanezumab. $74.4 \%$ ) occurred on the day of injection (Table 4). Only two participants on galcanezumab (galcanezumab $120 \mathrm{mg}, 1$ [1.4\%] and galcanezumab 240 mg, 1 [1.2\%]) had a reaction after 14 days. Majority of the injection-site reactions occurred on the day of injection and were resolved, either on the same day or a few days afterwards (mean [SD] duration in days, injection-site pain: placebo, 1.6 [8.35]; galcanezumab $120 \mathrm{mg}, 1.2$ [1.01]; galcanezumab $240 \mathrm{mg}, 1.5$ [2.68] and injection-site reaction excluding pain: placebo, 2.0 [8.76]; galcanezumab $120 \mathrm{mg}, 2.8$ [5.46]; galcanezumab $240 \mathrm{mg}, 2.7$ [4.17]).

\section{Injection-site reactions during long-term treatment with galcanezumab in REGAIN and study CGAJ}

During the long-term exposure of galcanezumab, 289 (289/1326, 21.8\%) participants reported AEs related to injection-sites. The most frequently reported injection-site reactions were injection-site pain (galcanezumab pooled, 108/1326 [8.1\%]), unspecified injection-site reaction (galcanezumab pooled, 103/1326 [7.8\%]), injection-site erythema (galcanezumab pooled, 62/1326 [4.7\%]), and injection-site pruritus (galcanezumab pooled, 30/1326 [2.3\%]; Table 5). Overall nine patients discontinued the treatment due to injection-site reactions (Study CGAJ, 
Table 2 Summary of injection-site reactions in pooled EVOLVE-1, EVOLVE-2, and REGAIN (placebo-controlled analysis set)

\begin{tabular}{|c|c|c|c|}
\hline & $\begin{array}{l}\text { Placebo } \\
(N=1451)\end{array}$ & $\begin{array}{l}\text { GMB } 120 \mathrm{mg} \\
(N=705)\end{array}$ & $\begin{array}{l}\text { GMB } 240 \mathrm{mg} \\
(N=730)\end{array}$ \\
\hline Any/all ISRs, n (\%) & $183(12.6)$ & $128(18.2)$ & $166(22.7)$ \\
\hline ISRs excluding pain, n (\%) & $60(4.1)$ & $70(9.9)$ & $106(14.5)$ \\
\hline \multicolumn{4}{|l|}{ ISRs, n (\%) } \\
\hline IS pain & $138(9.5)$ & $71(10.1)$ & 85 (11.6) \\
\hline Unspecified ISR & $14(1.0)$ & $22(3.1)$ & $45(6.2)$ \\
\hline IS erythema & $20(1.4)$ & $20(2.8)$ & $29(4.0)$ \\
\hline IS pruritus & $2(0.1)$ & $15(2.1)$ & $24(3.3)$ \\
\hline IS bruising & $9(0.6)$ & $4(0.6)$ & $10(1.4)$ \\
\hline IS swelling & $1(0.1)$ & $8(1.1)$ & $4(0.6)$ \\
\hline IS rash & $2(0.1)$ & $6(0.9)$ & $4(0.6)$ \\
\hline IS induration & $1(0.1)$ & $3(0.4)$ & $3(0.4)$ \\
\hline IS discomfort & $3(0.2)$ & $3(0.4)$ & $2(0.3)$ \\
\hline IS hematoma & $7(0.5)$ & $1(0.1)$ & $3(0.4)$ \\
\hline IS hypersensitivity & 0 & $1(0.1)$ & $3(0.4)$ \\
\hline IS mass & 0 & $3(0.4)$ & 0 \\
\hline IS haemorrhage & $2(0.1)$ & $1(0.1)$ & $1(0.1)$ \\
\hline IS inflammation & 0 & $1(0.1)$ & $1(0.1)$ \\
\hline IS irritation & $3(0.2)$ & $1(0.1)$ & $1(0.1)$ \\
\hline IS urticarial & $1(0.1)$ & $1(0.1)$ & $1(0.1)$ \\
\hline IS discoloration & 0 & 0 & $1(0.1)$ \\
\hline IS oedema & $1(0.1)$ & $1(0.1)$ & 0 \\
\hline IS papules & 0 & $1(0.1)$ & 0 \\
\hline IS vesicles & 0 & 0 & $1(0.1)$ \\
\hline IS warmth & $1(0.1)$ & 0 & 0 \\
\hline
\end{tabular}

Abbreviations: GMB galcanezumab, $n$ number of participants within each specific category, IS injection-site, ISR injection-site reaction, $N$ number of participants in the intent-to-treat population

Note: All values are for injection-site reactions during the double-blind treatment phase from study start up to 6 months for EVOLVE-1 and EVOLVE-2 and up to 3 months for REGAIN

$n=5$; REGAIN, $n=4$ ). All discontinuations were observed following multiple doses of galcanezumab (fourth, $n=1$; fifth injection, $\mathrm{n}=1$; sixth injection, $\mathrm{n}=1$; seventh injection, $n=3$; ninth injection, $\mathrm{n}=1$; 10th injection, $n=2$ ).

To evaluate if galcanezumab-treated patients reported multiple injection-site reactions (excluding pain) over consecutive monthly injections, the number of TEAEs related to injection-sites (excluding pain) by total number of doses is provided in Table 6 . In summary $81 \%$ of patients received 9 doses or more of galcanezumab and most patients reported 1 to 3 events with monthly injections over 9 to 12 months suggesting that the reporting of injection-site reactions (excluding pain) did not increase with multiple dose administrations.

\section{Discussion}

In Phase 3 studies demonstrating efficacy and safety of galcanezumab as a treatment option for management of migraine, injection-site reactions were the most commonly reported AEs. The incidence of injection-site pain was highest among all reported injection-site reactions and was reported equally by participants receiving galcanezumab and placebo. Unspecified injection-site reaction, injection-site erythema and injection-site pruritus were significantly $(P<$ 0.001 ) higher in participants being treated with galcanezumab compared with placebo. Overall, the incidence of injection-site reactions were higher in the galcanezumab dose groups $(240 \mathrm{mg}>120 \mathrm{mg}$ ) compared with placebotreated participants and appear to be driven by AEs related to injection-sites including unspecified injection-site reaction, injection-site erythema, and injection-site pruritus. These reactions are commonly observed with other approved monoclonal antibodies for subcutaneous use, including adalimumab, denosumab, ixekizumab, and canakinumab [16-18] as well as CGRP monoclonal antibodies [7, 19, 20].

In this post hoc analysis of four Phase 3 studies, we provide a more comprehensive summary of injectionsite reactions with short-term and long-term exposure 


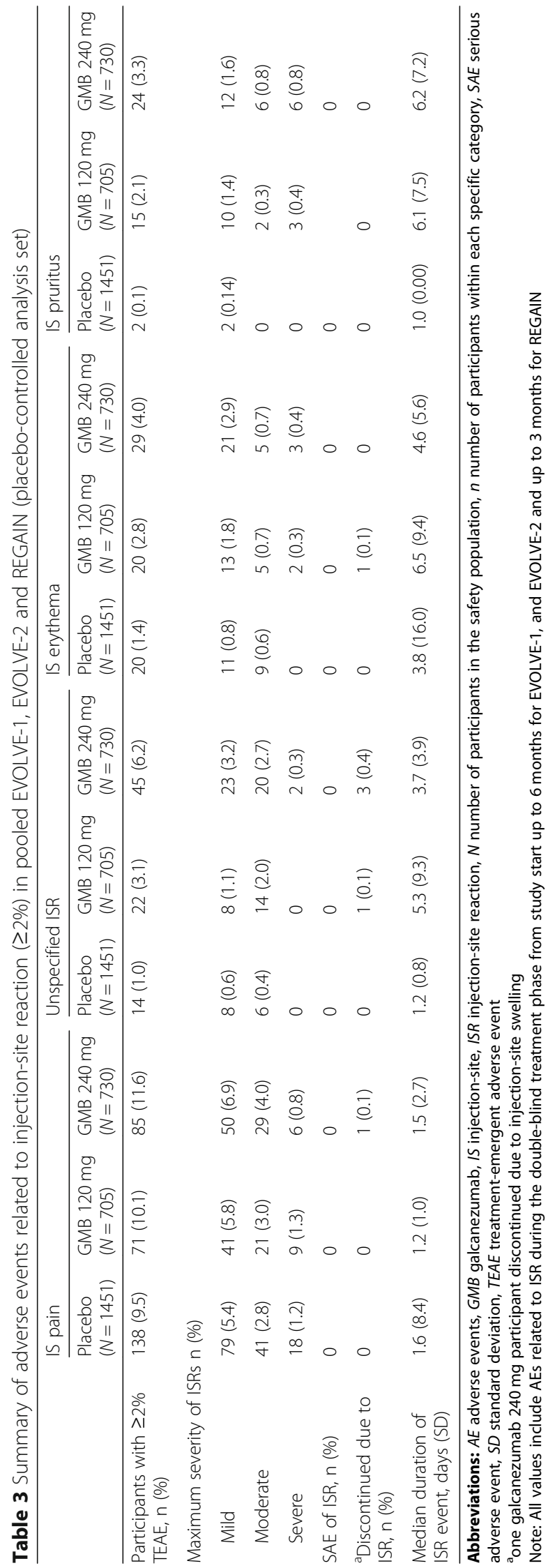




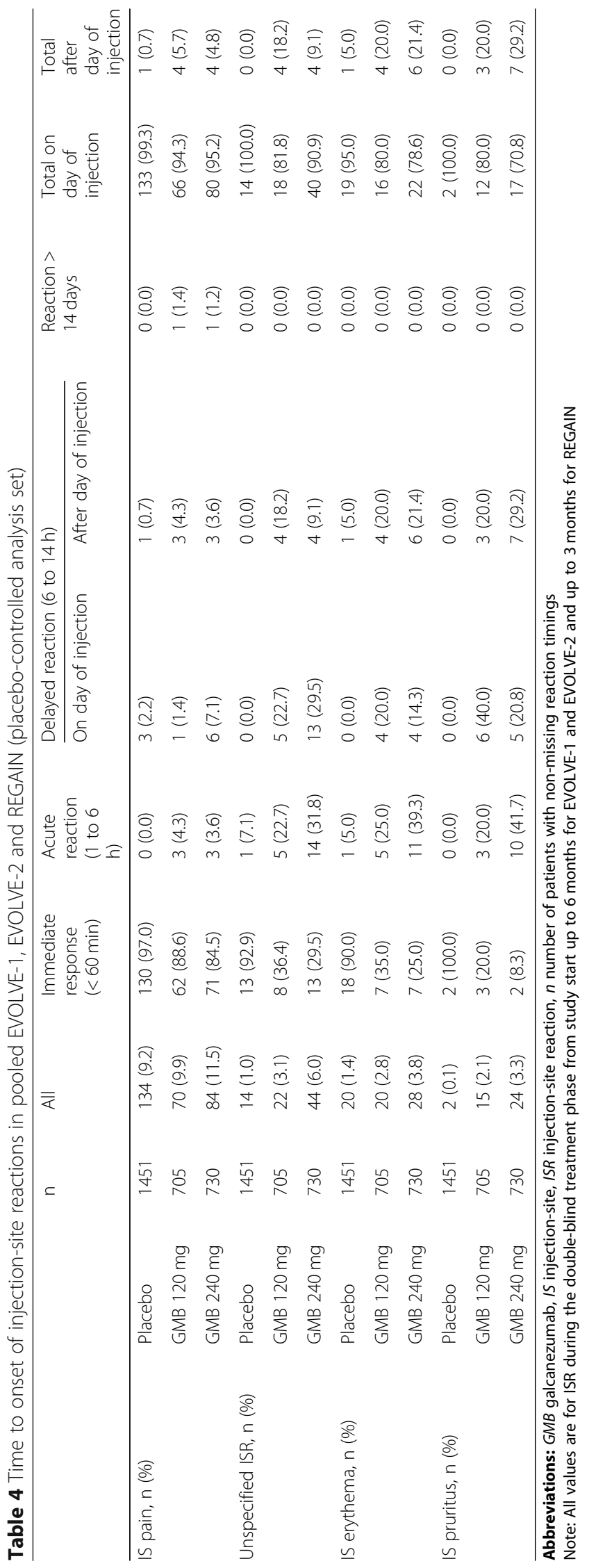


Table 5 Injection-site reactions by monthly dosing interval among pooled galcanezumab-treated participants in CGAJ and REGAIN studies, $\geq 1 \%$ incidence (galcanezumab exposure analysis set)

\begin{tabular}{ll}
\hline Participants with $\geq 1$ ISRs & Total, $\mathrm{n}(\%)$ \\
\hline $\mathbf{N}$ & 1326 \\
IS pain & $108(8.1)$ \\
Unspecified ISRs & $103(7.8)$ \\
IS erythema & $62(4.7)$ \\
IS pruritus & $30(2.3)$ \\
IS bruising & $25(1.9)$ \\
IS rash & $18(1.4)$ \\
IS hematoma & $14(1.1)$
\end{tabular}

Abbreviations: IS injection-site, ISR injection-site reaction, $N$ number of galcanezumab-treated participants, $n$ number of participants within each specific category

Note: All values are for ISRs from study start up to 12 months for REGAIN and CGAJ studies; pooled galcanezumab = galcanezumab

$120 \mathrm{mg}+$ galcanezumab $240 \mathrm{mg}$

to galcanezumab. Of the most commonly reported injection-site reactions in galcanezumab-treated participants, 70 to $100 \%$ were reported on the day of injection, were generally mild-to-moderate in severity, non-serious, and resolved spontaneously. In total 16/1705 (<1\%) treatment discontinuations were observed due to injection-site

Table 6 Summary of participants with single or multiple injection-site reactions excluding pain among pooled galcanezumab-treated participants in Study CGAJ and REGAIN (galcanezumab exposure analysis set)

\begin{tabular}{llll}
\hline Injection-site reactions, $\mathrm{n}(\%)$ & & & \\
\hline Total doses received $(\boldsymbol{N}=1326)$ & 1 to 3 & 4 to 9 & $>10$ \\
\hline $1(26)$ & $2(7.7)$ & 0 & 0 \\
$2(36)$ & $2(5.6)$ & 0 & 0 \\
$3(32)$ & $1(3.1)$ & $1(3.1)$ & 0 \\
$4(35)$ & $6(17.1)$ & 0 & 0 \\
$5(32)$ & $6(18.8)$ & 0 & 0 \\
$6(39)$ & $5(12.8)$ & $3(7.7)$ & 0 \\
$7(29)$ & $7(24.1)$ & $2(6.9)$ & 0 \\
$8(26)$ & $7(26.9)$ & 0 & 0 \\
9 (433) & $30(6.9)$ & $12(2.8)$ & $2(0.5)$ \\
$10(9)$ & $3(33.3)$ & $1(11.1)$ & 0 \\
$11(11)$ & $2(18.2)$ & 0 & 0 \\
$12(618)$ & $97(15.7)$ & $34(5.5)$ & $6(1.0)$ \\
\hline
\end{tabular}

athe number of patients receiving up to nine doses of galcanezumab, includes patients from both Study CGAJ (Month 0 to Month 12) and REGAIN (galcanezumab, Month 0 to Month 12; and placebo patients who had received placebo treatment from Month 0 to Month 3 and then initiated galcanezumab in the open-label extension phase and could receive up to nine doses of galcanezumab

Abbreviations: $N$ number of galcanezumab-treated participants with a specific number of galcanezumab doses, $n$ number of patients within each specific category reactions; seven were observed in placebo-controlled analysis set and nine (Study CGAJ, $n=5$; REGAIN, $n=4$ ) were observed in the galcanezumab exposure analysis set. Most participants reported onset of injection-site pain within $60 \mathrm{~min}$ of injection, and approximately $50 \%$ of injection-site pain was reported as injection-site burning. Of interest, $17.5 \%$ of patients reported a history of hypersensitivity, however only $5.2 \%$ of those patients reported injection-site related AEs other than pain. During exposure to galcanezumab up to 12 months of treatment, the number of injection-site reactions reported by the same patient does not increase with more doses received.

Notably, therapeutic monoclonal antibodies (mAb), including galcanezumab, have the potential to have antidrug antibody (ADA) formation, which in turn can block the active mAb site. The formation of ADA can be associated with changes to pharmacokinetic and pharmacodynamic parameters, resulting in a range of effects from no clinically important effects to reduced drug efficacy and/or increased risk of adverse events (AEs) [21, 22]. A recently published post hoc analysis of EVOLVE-1, EVOLVE-2, REGAIN and CGAJ studies, showed that approximately 2.6 to $12.4 \%$ of galcanezumab-treated patients developed treatment-emergent ADA. The characteristics of the immune response observed were not related to any clinically meaningful consequences on pharmacokinetic, pharmacodynamics, efficacy, or safety of galcanezumab. There were no differences in injectionsite reactions reported in patients who developed treatment emergent ADA compared to those who did not.

Therapeutic proteins are typically administered in the subcutaneous tissue, especially when the treatment is required frequently, on a long-term basis and requires self-administration [23]. Injection-site reactions with therapeutic proteins may arise from participant-related factors such as variability in injection speed (association of injection-site pain with fast injections), participant-toparticipant differences in pain tolerance. The injectionsite reactions with therapeutic proteins may also arise from variations in formulation-related factors such as formulation temperature (which should ideally be close to body temperature), type of injectable device used (prefilled syringe versus autoinjector), injection volume (ideally should be $\leq 3 \mathrm{~mL}$ [24]), $\mathrm{pH}$ (ideally should be physiological) and excipients [23, 25]. For galcanezumab, the $\mathrm{pH}$ of the formulation is between 5.3 and 6.3, the injection volume is $1 \mathrm{~mL}$, the inactive excipient contains polysorbate 80, L-histidine, histidine hydrochloride monohydrate, and sodium chloride [26]. During shortterm exposure to galcanezumab, we observed similar incidence of injection-site pain between placebo- and galcanezumab-treated participants. This may explain the possibility that formulation-based factors, such as nonphysiological $\mathrm{pH}$ (i.e. <7) and presence of polysorbate 
80 could have resulted in injection-site pain. These findings are in agreement with the earlier findings of Kaiser et al. (2012), who have cited injection-site pain associated with Kineret (anakinra) owing to its non-physiological $\mathrm{pH}$ and presence of polysorbate 80 in the formulation [25, 27].

This post hoc analysis, which was not pre-specified at the time of design of the EVOLVE-1, EVOLVE-2, REGAIN and Study CGAJ, limits its ability to make definitive conclusions. There could also be some under-reporting possibly as all AEs were reported spontaneously by the study participants, and use of analgesic, and histamine creams, and potential comfort measures were not excluded. Lastly, galcanezumab injections were administered by study site personnel in the galcanezumab Phase 3 studies, with the exception of Study CGAJ. In Study CGAJ, patients self-administered galcanezumab either with the prefilled syringe or the autoinjector devices. The injection experience and tolerability of self-administration with both devices has been published elsewhere [15].

\section{Conclusions}

In conclusion, galcanezumab-treated participants reported a significantly higher frequency of injection-site reactions versus placebo. Most of these events were self-limiting; no SAEs related to injection-site were reported in any of the Phase 3 migraine studies. Future post-marketing study evidence is warranted to augment these findings.

\section{Supplementary information}

Supplementary information accompanies this paper at https://doi.org/10. 1186/s12883-020-01775-4.

Additional file 1. List of ethics committee.

\section{Abbreviations}

ADA: Anti-drug antibody; AE: Adverse events; CGRP: Calcitonin Gene-Related Peptide; DB: Double-blind; HLT: High-level term; OL: Open-label; OLE: Openlabel extension; PT: Preferred term; SAEs: Serious adverse events; SD: Standard deviation

\section{Acknowledgements}

None.

\section{Authors' contributions}

VLS provided substantial contribution for the study conception and design, acquisition of data and interpretation of data. SW provided substantial contribution for the analysis and interpretation of data. JB and BK provided substantial contribution for the acquisition of data. RB provided substantial contribution for the interpretation of data and in drafting the article. AC and KAD provided substantial contribution towards interpretation of data. All authors were involved in drafting/revising the article for important intellectual content and have given final approval of the version to be published.

\section{Funding}

Eli Lilly and Company funded the studies in whole and its employees and assigns were involved in study design, data collection, data analysis, data interpretation, and writing of all related reports and publications. The corresponding author had full access to all study data and had final responsibility for the decision to submit for publication.

\section{Availability of data and materials}

Lilly provides access to all individual participant data collected during the trial, after anonymization, with the exception of pharmacokinetic or genetic data. Data are available to request 6 months after the indication studied has been approved in the US and EU and after primary publication acceptance, whichever is later. No expiration date of data requests is currently set once data are made available. Access is provided after a proposal has been approved by an independent review committee identified for this purpose and after receipt of a signed data sharing agreement. Data and documents, including the study protocol, statistical analysis plan, clinical study report, blank or annotated case report forms, will be provided in a secure data sharing environment. For details on submitting a request, see the instructions provided at www.viviliorg.

\section{Ethics approval and consent to participate}

The studies in this integrated safety analysis were conducted in concordance with the ethical principles that have their origin in the Declaration of Helsinki guidelines. All patients provided written informed consent before study participation.

The study protocols, for all four studies, were reviewed and approved by the Institutional Review Board, Medical Ethics Committee or Medical Research \& Ethics Committee of the participating study sites (Additional file 1).

\section{Consent for publication}

Not applicable.

\section{Competing interests}

Eli Lilly and Company funded the studies in whole. VLS, SW, RB, KAD and AC reported being full-time employee of Eli Lilly and Company. SW and AC also report being minor shareholders of Eli Lilly and Company. JB reported receiving ancillary supplies, clinical grant, food and beverage, lodging, transportation and travel grant from Eli Lilly and company. BK reports receiving personal fees from Eli Lilly and Company, TEVA, Novartis, Sanofi Korea, Allergan Korea, Lundbeck Kora, SK chemical, GSK Korea.

\section{Author details}

${ }^{1}$ Lilly Research Laboratories, Lilly Corporate Center, Indianapolis, IN, USA. ${ }^{2} \mathrm{St}$ Louis University Hospital, St Louis, USA. ${ }^{3}$ Nowon Eulji Medical Center, Seoul, Republic of Korea. ${ }^{4}$ Eli Lilly Services India Private Limited, Bangalore, India. ${ }^{5}$ Eli Lilly Italia, Sesto Fiorentino, Italy.

Received: 15 February 2020 Accepted: 10 May 2020 Published online: 19 May 2020

\section{References}

1. Goadsby PJ, Edvinsson L, Ekman R. Vasoactive peptide release in the extracerebral circulation of humans during migraine headache. Ann Neurol. 1990;28:183-7.

2. Goadsby PJ, Edvinsson L, Ekman R. Release of vasoactive peptides in the extracerebral circulation of humans and the cat during activation of the trigeminovascular system. Ann Neurol. 1988;23:193-6.

3. Lassen LH, Haderslev PA, Jacobsen VB, Iversen HK, Sperling B, Olesen J. CGRP may play a causative role in migraine. Cephalalgia. 2002;22:54-61.

4. Stauffer VL, Dodick DW, Zhang Q, Carter JN, Ailani J, Conley RR. Evaluation of Galcanezumab for the prevention of episodic migraine: the EVOLVE-1 randomized clinical trial. JAMA Neurol. 2018;75:1080-8.

5. The Lancet Neurology. Complicated decisions on new migraine-prevention therapies. Lancet Neurol. 2019;18:221.

6. Bangs ME, Kudrow D, Wang S, Oakes TM, Terwindt GM, Magis D, YunesMedina L, Stauffer VL. Safety and tolerability of monthly galcanezumab injections in patients with migraine: integrated results from migraine clinical studies. BMC Neurol. 2020;20:25.

7. Silberstein SD, McAllister $P$, Ning $X$, Faulhaber N, Lang N, Yeung $P$, Schiemann J, Aycardi E, Cohen JM, Janka L, et al. Safety and tolerability of Fremanezumab for the prevention of migraine: a pooled analysis of phases $2 b$ and 3 clinical trials. Headache. 2019;59:880-90.

8. Ashina M, Kudrow D, Reuter U, Dolezil D, Silberstein S, Tepper SJ, Xue F, Picard $H$, Zhang F, Wang A, et al. Long-term tolerability and nonvascular safety of erenumab, a novel calcitonin gene-related peptide receptor antagonist for prevention of migraine: A pooled analysis of four placebo- 
controlled trials with long-term extensions. Cephalalgia. 2019;39(14):1798808.

9. Hay J. Complications at site of injection of depot neuroleptics. BMJ. 1995:311:421.

10. Murdaca G, Spano F, Puppo F. Selective TNF-alpha inhibitor-induced injection site reactions. Expert Opin Drug Saf. 2013;12:187-93.

11. Skljarevski V, Matharu M, Millen BA, Ossipov MH, Kim BK, Yang JY. Efficacy and safety of galcanezumab for the prevention of episodic migraine: results of the EVOLVE-2 phase 3 randomized controlled clinical trial. Cephalalgia. 2018;38:1442-54

12. Detke HC, Goadsby PJ, Wang S, Friedman DI, Selzler KJ, Aurora SK Galcanezumab in chronic migraine: the randomized, double-blind, placebocontrolled REGAIN study. Neurology. 2018;91:e2211-e21.

13. Ford JH, Ayer DW, Zhang Q, Carter JN, Leroux E, Skljarevski V, Aurora SK, Tockhorn-Heidenreich A, Lipton RB. Two randomized migraine studies of galcanezumab: effects on patient functioning and disability. Neurology. 2019;93:e508-e17.

14. Camporeale A, Kudrow D, Sides R, Wang S, Van Dycke A, Selzler K, Stauffer VL. A phase 3, long-term, open-label safety study of Galcanezumab in patients with migraine. BMC Neurol. 2018;18:188.

15. Stauffer VL, Sides R, Lanteri-Minet M, Kielbasa W, Jin Y, Selzler KJ, Tepper SJ. Comparison between prefilled syringe and autoinjector devices on patientreported experiences and pharmacokinetics in galcanezumab studies. Patient Prefer Adherence. 2018;12:1785-95.

16. FDA Labelling Packaging Insert: Prescribing informtaion: HUMIRA ${ }^{\oplus}$ (adalimumab) injection, updated January 2019. https://www.rxabbvie.com/ pdf/humira.pdf. Accessed on 17 Sep 2019.

17. FDA Labelling Packaging Insert: Prescribing informtaion: Prolia ${ }^{\odot}$ (denosumab), updated July 2019. https:/www.pi.amgen.com/ /media/ amgen/repositorysites/pi-amgen-com/prolia/prolia_pi.pdf. Accessed on 17 Sep 2019.

18. FDA Labelling Packaging Insert: Prescribing informtaion: ILARIS (canakinumab), updated July 2019. https://www.accessdata.fda.gov/ drugsatfda_docs/label/2012/125319s047/bl.pdf. Accessed on 17 Sep 2019.

19. Analysis of injection site reactions across four placebo controlled trials of erenumab for migraine prevention. 11th European Headache Federation Congress jointly with 31st Congress of the Italian Society for the Study of Headaches: Rome, Italy. 01-03 December 2017. J Headache Pain. 2017;18:111

20. Shear NH, Paul C, Blauvelt A, Gooderham M, Leonardi C, Reich K, Ohtsuki M, Pangallo B, Xu W, Ball S, et al. Safety and tolerability of Ixekizumab: integrated analysis of injection-site reactions from 11 clinical trials. J Drugs Dermatol. 2018;17:200-6.

21. Martinez JM, Garce S, Anglin G, Hodsdon M, Kielbasa W, Moser BA, Pearlman $E$. Immunogenicity findings from Phase 3 galcanezumab trials in patients with episodic or chronic migraine (Abstract number P25). Presented at the $12^{\text {th }}$ European Headache Federation Congress jointly with $32^{\text {nd }}$ National Congress of the Italian Society for the Study of Headaches, 28-30 September 2018, Florence, Italy. J Headache Pain. 2018;19:80.

22. Martinez JM, Hindiyeh N, Anglin G, Kalidas K, Hodsdon ME, Kielbasa W, Moser BA, Pearlman EM, Garces S. Assessment of immunogenicity from galcanezumab phase 3 trials in patients with episodic or chronic migraine. Cephalalgia. 2020;0:333102420920642.

23. Mathaes R, Koulov A, Joerg S, Mahler HC. Subcutaneous injection volume of biopharmaceuticals-pushing the boundaries. J Pharm Sci. 2016;105:2255-9.

24. Berteau C, Filipe-Santos O, Wang T, Rojas HE, Granger C, Schwarzenbach F. Evaluation of the impact of viscosity, injection volume, and injection flow rate on subcutaneous injection tolerance. Med Devices (Auckl). 2015:8:473-84

25. Kaiser C, Knight A, Nordstrom D, Pettersson T, Fransson J, Florin-Robertsson E, Pilstrom B. Injection-site reactions upon Kineret (anakinra) administration: experiences and explanations. Rheumatol Int. 2012;32:295-9.

26. FDA Labelling Packaging Insert: Prescribing informtaion: Emgality (Galcanezumab), updated 2019. https://www.accessdata.fda.gov/drugsatfda_ docs/label/2019/761063s003lbl.pdf. Accessed on 15 July 2019.

27. Schwartzberg LS, Navari RM. Safety of Polysorbate 80 in the oncology setting. Adv Ther. 2018;35:754-67.

\section{Publisher's Note}

Springer Nature remains neutral with regard to jurisdictional claims in published maps and institutional affiliations.

\section{Ready to submit your research? Choose BMC and benefit from:}

- fast, convenient online submission

- thorough peer review by experienced researchers in your field

- rapid publication on acceptance

- support for research data, including large and complex data types

- gold Open Access which fosters wider collaboration and increased citations

- maximum visibility for your research: over $100 \mathrm{M}$ website views per year

At BMC, research is always in progress.

Learn more biomedcentral.com/submissions 